\title{
Comparison of large-scale dynamical variability in the extratropical stratosphere among the JRA-55 family data sets: impacts of assimilation of observational data in JRA-55 reanalysis data
}

\author{
Masakazu Taguchi \\ Department of Earth Science, Aichi University of Education, Kariya, 448-8542, Japan \\ Correspondence to: Masakazu Taguchi (mtaguchi@auecc.aichi-edu.ac.jp) \\ Received: 1 April 2017 - Discussion started: 2 May 2017 \\ Revised: 28 July 2017 - Accepted: 8 August 2017 - Published: 21 September 2017
}

\begin{abstract}
This study compares large-scale dynamical variability in the extratropical stratosphere, such as major stratospheric sudden warmings (MSSWs), among the Japanese 55year Reanalysis (JRA-55) family data sets. The JRA-55 family consists of three products: a standard product (STDD) of the JRA-55 reanalysis data and two sub-products of JRA55C (CONV) and JRA-55AMIP (AMIP). CONV assimilates only conventional surface and upper-air observations without assimilation of satellite observations, whereas AMIP runs the same numerical weather prediction model without assimilation of observational data. A comparison of the occurrence of MSSWs in Northern Hemisphere (NH) winter shows that, compared to STDD, CONV delays several MSSWs by 1 to 4 days and also misses a few MSSWs. CONV also misses the Southern Hemisphere (SH) MSSW in September 2002. AMIP shows significantly fewer MSSWs in Northern Hemisphere winter and especially lacks MSSWs of the high aspect ratio of the polar vortex in which the vortex is highly stretched or split. A further examination of daily geopotential height differences between STDD and CONV reveals occasional peaks in both hemispheres that are separated from MSSWs. The delayed and missed MSSW cases have smaller height differences in magnitude than such peaks. The height differences for those MSSWs include large contributions from the zonal component, which reflects underestimations in the weakening of the zonal mean polar night jet in CONV. We also explore strong planetary wave forcings and associated polar vortex weakenings for STDD and AMIP. We find a lower frequency of strong wave forcings and weaker vortex responses to such wave forcings in AMIP, consistent with the lower MSSW frequency.
\end{abstract}

\section{Introduction}

Large-scale dynamical variability is an important feature in the extratropical stratosphere, especially in the Northern Hemisphere (NH) winter stratosphere (e.g., Labitzke and van Loon, 1999; Yoden et al., 2002; Waugh and Polvani, 2010). The NH winter stratosphere exhibits large intraseasonal and interannual variations, reflecting anomalously strong and weak conditions of the polar vortex. The polar vortex in the Southern Hemisphere (SH) spring stratosphere also shows large variations, e.g., in its strength and distribution, while the SH winter stratosphere is more dynamically quiescent.

Some weak conditions of the polar vortex correspond to the occurrence of stratospheric sudden warmings (SSWs), during which the polar night jet weakens and polar stratospheric temperatures rise as the polar vortex largely distorts and/or breaks down (e.g., Limpasuvan et al., 2004; Charlton and Polvani, 2007). An SSW is classified as a major SSW (MSSW) when it accompanies a reversal of the zonal mean zonal wind often seen at $60^{\circ} \mathrm{N}, 10 \mathrm{hPa}$ (e.g., Butler et al., 2015). An SSW without such a zonal wind reversal is classified as a minor SSW.

Previous studies investigate aspects of such dynamical variability in the extratropical stratosphere using multiple reanalysis data sets. Reanalysis data sets are a vital tool to understand atmospheric variability and relevant processes in the climate science including middle atmosphere dynamics, but different reanalyses sometimes yield different results for the same diagnostics. Martineau and Son (2010) examined time evolutions of the Northern Annular Mode index for stratospheric vortex weakening and intensification events among five reanalyses and found good agreement. Martineau 
et al. (2016) investigated dynamical consistency in the extratropical stratosphere among eight reanalyses as quantified by the residual of the zonal momentum equation. Applying a multiple linear regression analysis to nine reanalyses, Mitchell et al. (2015) studied signatures of interannual variability associated with natural forcings and found remarkable similarity among the data sets. Manney et al. (2005) conducted a diagnostic comparison of the SH MSSW in September 2002 among several meteorological data sets.

Some of these studies are part of a coordinated activity of the Stratosphere-troposphere Processes And their Role in Climate (SPARC) Reanalysis Intercomparison Project (SRIP; Fujiwara et al., 2017). The climatology and interannual variability of monthly mean temperature and wind fields are surveyed in the S-RIP framework. Furthermore, various aspects of the dynamical coupling between the NH extratropical stratosphere and troposphere will also be investigated there.

The present study focuses on the Japanese 55-year Reanalysis (JRA-55; Kobayashi et al., 2015) data among others, which is one of newer reanalyses. A unique feature of JRA55 is that, in addition to the standard product (STDD), two companion products, JRA-55C (CONV) and JRA-55AMIP (AMIP), are also available. CONV assimilates conventional surface and upper-air observations only, without assimilation of satellite observations. AMIP is an AMIP-type simulation using the same forecast model as in STDD and CONV, without assimilation of any observational data. The three products are called the "JRA-55 family data sets" as a whole (Kobayashi et al., 2014).

Previous studies investigated some aspects of the JRA-55 family data sets, especially in the stratosphere. Kobayashi and Iwasaki (2016) examined the Brewer-Dobson circulation in the lower stratosphere to show that the mass stream function at $100 \mathrm{hPa}$ (vertically integrated northward mass flux above $100 \mathrm{hPa}$ ) is similar between STDD and CONV in annual and seasonal averages, but it is much weaker for AMIP. Kobayashi et al. (2014) and Kobayashi and Iwasaki (2016) also showed that the polar night jet in the winter stratosphere for each hemisphere is stronger for CONV than for STDD, which is associated with weaker upward wave propagation and driving. The differences of AMIP from STDD are qualitatively similar but are much larger in magnitude.

This study seeks to compare the climatology and largescale dynamical variability, such as frequency and vortex geometry of SSWs, in the NH and SH extratropical stratosphere among the JRA-55 family data sets. A motivation for this study is that dynamical variability in the extratropical stratosphere remains relatively unexplored in the JRA-55 family data sets, while they provide a good opportunity for a clear comparison owing to their meticulous design. In order to better understand the differences of MSSWs, we further describe differences of daily geopotential height fields between STDD and CONV and relate them to the occurrence of MSSWs. We also explore strong wave forcings and associated stratospheric vortex responses for STDD and AMIP.

The rest of the paper is organized as follows. Section 2 explains the data and analysis methods used in this study. Section 3 surveys the climatology and variability in the extratropical stratosphere in the JRA-55 family data sets. Section 4 further examines STDD and CONV, and Sect. 5 examines STDD and AMIP. Finally, Sect. 6 provides the summary and discussion.

\section{Data and analysis method}

\subsection{Data}

This study makes use of daily averages for the three products of the JRA-55 family data sets. The horizontal resolution is $2.5^{\circ} \times 2.5^{\circ}$, with 37 levels up to $1 \mathrm{hPa}$. The full period is from 1958 to 2012, but we use a shorter period in some comparisons (see below). We mainly use the zonal mean zonal wind, poleward eddy heat flux by wave components of zonal wave numbers 1-3 (waves 1-3), and geopotential height. The eddy heat flux in the extratropical lower stratosphere (e.g., $40-90^{\circ} \mathrm{N}, 100 \mathrm{hPa}$ ) is used as a proxy for planetary wave forcing from the troposphere, since it is proportional to the vertical component of the Eliassen-Palm (EP) flux under the quasi-geostrophic scaling (Andrews et al., 1987).

We regard STDD, or the standard JRA-55 reanalysis data (Kobayashi et al., 2015), as a good representation of the real world. A comparison of CONV to STDD elucidates the effects of assimilation of satellite data, since the inclusion or exclusion of assimilation of satellite data is the only difference between the two (Kobayashi et al., 2014). We also compare AMIP to STDD to examine model biases, since the AMIP data are obtained from an AMIP-type forecast simulation using the same numerical weather prediction model and same boundary conditions (sea surface temperatures, greenhouse gases, etc.; see Kobayashi et al., 2015) without assimilation of any observational data.

For the comparison between STDD and CONV, we use the data from 1972-1973 to 2012-2013 when CONV is available. For the comparison between STDD and AMIP, we use the data from 1957-1958 to 2012-2013 (i.e., full period). We sort the data in time for both hemispheres so that they begin from June in each year and end in May in the next year to facilitate our main focus on $\mathrm{NH}$ winter when the $\mathrm{NH}$ extratropical stratosphere is dynamically active. For both $\mathrm{NH}$ and $\mathrm{SH}$, we refer to each year from June to May by the year to which the month of January belongs.

\subsection{Analysis methods}

\subsubsection{Identification of MSSW onset dates}

The method outlined by Charlton and Polvani (2007) is basically followed to identify MSSWs. This method identifies 
Table 1. Onset dates of DJF MSSWs for STDD and CONV, with differences in the onset dates (number of days) of CONV from STDD. Empty cells indicate the period when CONV is unavailable. The "M" letters mean that the MSSWs identified in STDD are missed in CONV.

\begin{tabular}{|c|c|c|}
\hline STDD & $\mathrm{CONV}$ & DIFF \\
\hline 30 Jan 1958 & & \\
\hline 17 Jan 1960 & & \\
\hline 30 Jan 1963 & & \\
\hline 18 Dec 1965 & & \\
\hline 23 Feb 1966 & & \\
\hline 7 Jan 1968 & & \\
\hline 2 Jan 1970 & & \\
\hline 18 Jan 1971 & & \\
\hline 31 Jan 1973 & 1 Feb 1973 & 1 \\
\hline 9 Jan 1977 & 13 Jan 1977 & 4 \\
\hline 22 Feb 1979 & 22 Feb 1979 & 0 \\
\hline 29 Feb 1980 & 29 Feb 1980 & 0 \\
\hline 6 Feb 1981 & $\mathrm{M}$ & - \\
\hline 4 Dec 1981 & $\mathrm{M}$ & - \\
\hline 24 Feb 1984 & 24 Feb 1984 & 0 \\
\hline 1 Jan 1985 & 4 Jan 1985 & 3 \\
\hline 23 Jan 1987 & 23 Jan 1987 & 0 \\
\hline 8 Dec 1987 & 8 Dec 1987 & 0 \\
\hline 21 Feb 1989 & 21 Feb 1989 & 0 \\
\hline 15 Dec 1998 & 16 Dec 1998 & 1 \\
\hline 26 Feb 1999 & 26 Feb 1999 & 0 \\
\hline 11 Feb 2001 & 11 Feb 2001 & 0 \\
\hline 31 Dec 2001 & $\mathrm{M}$ & - \\
\hline 18 Jan 2003 & 18 Jan 2003 & 0 \\
\hline 5 Jan 2004 & 7 Jan 2004 & 2 \\
\hline 21 Jan 2006 & 22 Jan 2006 & 1 \\
\hline 24 Feb 2007 & 24 Feb 2007 & 0 \\
\hline 22 Feb 2008 & 22 Feb 2008 & 0 \\
\hline 24 Jan 2009 & 25 Jan 2009 & 1 \\
\hline 9 Feb 2010 & 9 Feb 2010 & 0 \\
\hline
\end{tabular}

the onset date (denoted as lag $=0$ day) of an MSSW as when the zonal mean zonal wind at $60^{\circ} \mathrm{N}, 10 \mathrm{hPa}$, reverses from a westerly wind to an easterly wind. We focus on MSSWs during the winter period of December-January-February (DJF). In order to identify two (or more) MSSWs in one season, the onset dates between two successive events must be separated more than 20 days, and the zonal wind must recover above $20 \mathrm{~m} \mathrm{~s}^{-1}$ between them. The latter condition is added to ensure that the polar vortex is sufficiently re-established after the first event. The resultant onset dates of the MSSWs identified for STDD and CONV are shown in Table 1. The onset dates for STDD are identical to those in Butler et al. (2017) as far as the DJF MSSWs are concerned.

\subsubsection{Calculation of polar vortex geometry}

In order to characterize the geometry of the polar vortex, centroid latitude (CL) and aspect ratio (AR) are calculated for the $10 \mathrm{hPa}$ height according to Seviour et al. (2013). This method diagnoses where the center of the vortex is located (CL) and how stretched the vortex is (AR), where the vortex is defined as the region of the $10 \mathrm{hPa}$ height lower than a threshold of $\mathrm{Zb}$. The parameter $\mathrm{Zb}$ is taken as the climatological and zonal mean of the $10 \mathrm{hPa}$ height at $60^{\circ} \mathrm{N}$ in each product and is different among the three products. The height fields are smoothed with a 5-day running mean in calculating CL and AR so as to filter out day-to-day fluctuations and capture dominant geometry features.

\subsubsection{Calculation of RMSD of geopotential height fields}

Differences in daily geopotential height fields, e.g., at $10 \mathrm{hPa}$, between STDD and CONV are evaluated with the root mean square difference (RMSD) as follows:

$\mathrm{RMSD}=\left[\frac{\sum_{i=1}^{n} w_{i}\left\{Z_{\mathrm{CONV}}\left(x_{i}\right)-Z_{\mathrm{STDD}}\left(x_{i}\right)\right\}^{2}}{\sum_{i=1}^{n} w_{i}}\right]^{1 / 2}$.

Here, $Z_{\text {STDD }}$ and $Z_{\mathrm{CONV}}$ denote geopotential height fields at an arbitrary level on a day of interest for STDD and CONV, respectively; $x_{i}$ denotes spatial grid points (longitude and latitude); and $w_{i}$ is the cosine of latitude. The summations are taken for all extratropical grid points (indexed with $i$ from 1 to $n$ ) poleward of $30^{\circ} \mathrm{N} / \mathrm{S}$. Note that an arbitrary RMSD value is divided into contributions from the zonal and wave components as follows:

$\mathrm{RMSD}^{2}=\mathrm{RMSD}_{\text {zonal }}^{2}+\mathrm{RMSD}_{\text {wave }}^{2}$

Here, $\mathrm{RMSD}_{\text {wave }}$ is calculated by applying Eq. (1) to wave fields of $Z_{\mathrm{STDD}}$ and $Z_{\mathrm{CONV}}$.

\section{Survey of climatology and variability in the extratropical stratosphere during $\mathrm{NH}$ winter and SH spring}

This section surveys the climatology (long-term mean) and variability in the extratropical stratosphere for $\mathrm{NH}$ winter and SH spring using the zonal mean zonal wind and $10 \mathrm{hPa}$ geopotential height.

\subsection{Climatology}

Figure 1 shows the climatological zonal mean zonal wind in DJF and September-October-November (SON) for STDD, CONV, and AMIP. Color shades plot only differences of CONV or AMIP from STDD that are judged to be statistically significant according to a Student's $t$ test (two-sided test) at the $95 \%$ confidence level. When taking differences between STDD and CONV, the STDD data after the 19721973 season are used as stated in Sect. 2.1. The degrees of 
(a) STDD, DJF
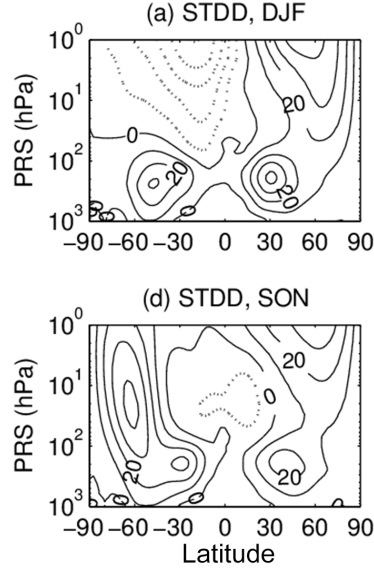

(b) CONV, DJF

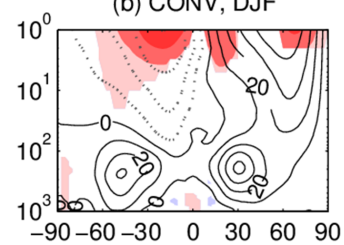

(e) CONV, SON

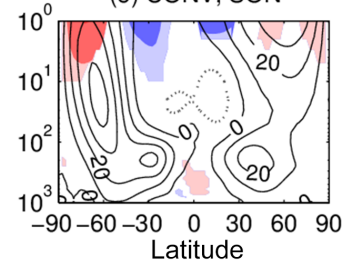

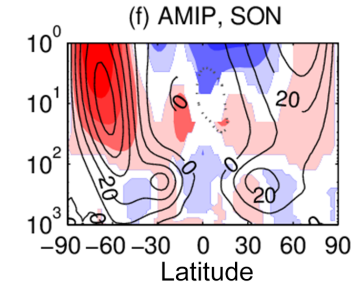

(c) AMIP, DJF

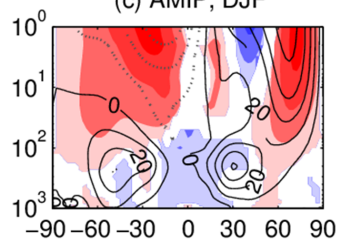

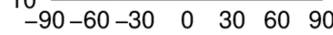

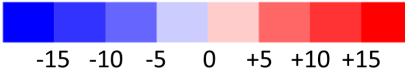

Figure 1. The climatological zonal mean zonal wind in the JRA-55 family data sets for (a-c) DJF and (d-f) SON in black contours. Panels (a, d) are for STDD, (b, e) for CONV, and (c, f) for AMIP. The contour interval is $10 \mathrm{~m} \mathrm{~s}^{-1}$. Panels (b, $\left.\mathbf{c}, \mathbf{e}, \mathbf{f}\right)$ also plot differences from STDD using color shades (see the color bar) that are statistically significant at the 95\% level. When taking differences of CONV from STDD, the STDD data after the 1972-1973 season are used. PRS is pressure.

(a) STDD, DJF

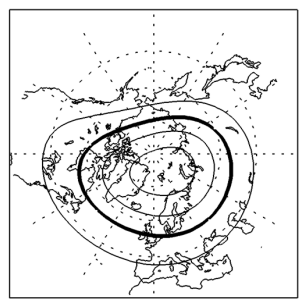

(d) STDD, SON

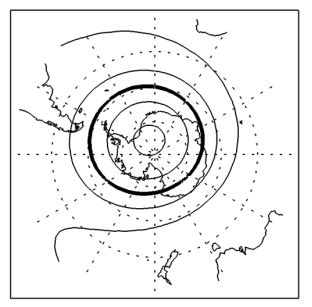

(b) CONV, DJF

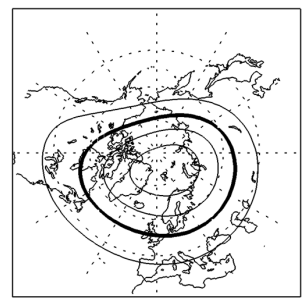

(e) CONV, SON

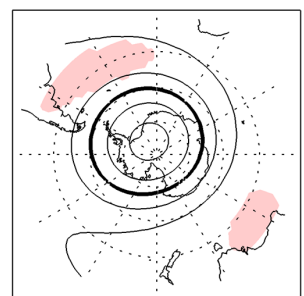

(c) AMIP, DJF

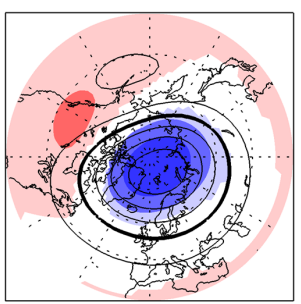

(f) AMIP, SON

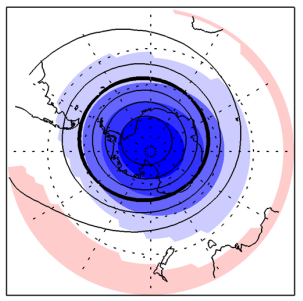

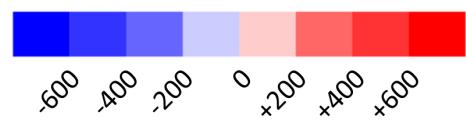

Figure 2. Same as in Fig. 1, but for the climatological mean $10 \mathrm{hPa}$ height. The contour interval is $500 \mathrm{~m}$. Thick contours denote $30000 \mathrm{~m}$.

freedom in the test are equated to the number of years, as the test uses DJF or SON means for the target years.

The climatological zonal wind is similar between STDD and CONV in a large part of the domain below the middle stratosphere for both seasons, although some differences are notable in the upper stratosphere. The differences are positive near the westerly jets in both hemispheres during the cold seasons, which indicate the stronger westerly jets for CONV.
The SH easterly winds for DJF also have positive differences. Negative differences appear in NH subtropical latitudes and SH midlatitudes for SON. Tropospheric wind differences are generally small in magnitude.

The wind differences of AMIP from STDD are roughly similar to those of CONV in spatial pattern but are larger in vertical extent and magnitude in the stratosphere. AMIP also shows significant differences in the troposphere, although 

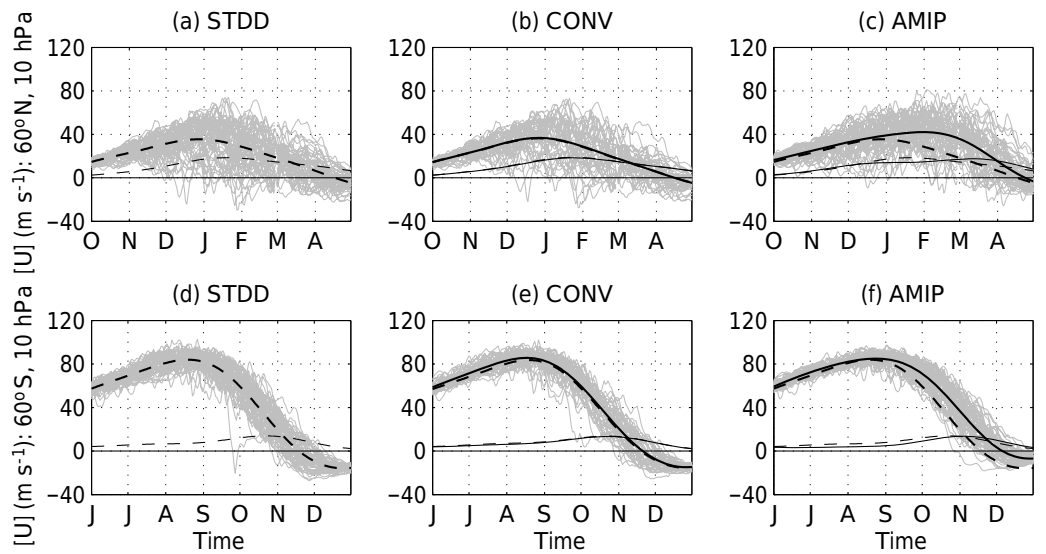

Figure 3. Daily time series of the zonal mean zonal wind $[U]$ at $10 \mathrm{hPa}$ (gray solid lines): (a-c) $60^{\circ} \mathrm{N}$ and $(\mathbf{d}-\mathbf{f}) 60^{\circ} \mathrm{S}$. The square brackets denote the zonal mean. Panels (a, d) are for STDD, (b, e) for CONV, and (e, f) for AMIP. The climatological seasonal cycle and standard deviation of interannual variability for each day are denoted by black broken lines in (a, c) and by black solid lines in the other panels. Broken lines in (b, c, e, f) denote the results from STDD. The STDD data after the 1972-1973 season are used in (b, e). Month labels are placed at the 1 st day of each month.

their magnitudes are smaller. In particular, the zonal wind has positive differences around $60^{\circ} \mathrm{N}$ for DJF, which extend to the stratosphere.

These differences in the polar night jet are consistently reflected in Fig. 2, which similarly shows maps of the climatological $10 \mathrm{hPa}$ geopotential height. The polar vortex is very similar between STDD and CONV in strength and shape for both $\mathrm{NH}$ winter and SH spring (Fig. 2b, e). This feature corresponds to the absence of significant differences in the zonal wind at $10 \mathrm{hPa}$ (Fig. 1b, e). On the other hand, AMIP simulates the vortex that is stronger than the STDD counterpart for both seasons (Fig. 2c, f), consistent with the positive wind differences around the polar night jet from the geostrophic wind relationship (Fig. 1c, f). Positive height differences are also notable in surrounding midlatitudes.

Some of these results about the climatological zonal wind are consistently seen in Kobayashi et al. (2014) and Kobayashi and Iwasaki (2016). The latter study further claimed that the stronger polar vortex in CONV and AMIP reflects weaker wave propagation and driving.

\subsection{Variability}

Next, we examine variability in the extratropical stratosphere by looking at daily time series of the zonal mean zonal wind at $60^{\circ} \mathrm{N} / \mathrm{S}, 10 \mathrm{hPa}$ (Fig. 3). The zonal mean zonal wind at this location is used as a measure of strength (and also flow direction) of the polar vortex for cold seasons in each hemisphere. The climatological seasonal cycle (long-term mean) and variability (standard deviation of interannual variability for each day) are also plotted in the figure. The climatology and standard deviation are smoothed in time so that they consist of low frequency components with periods longer than about 100 days.
The climatology and standard deviation of the zonal wind overlap between STDD and CONV for both $\mathrm{NH}$ and $\mathrm{SH}$ (Fig. 3b, e). A comparison of probability distribution functions (PDFs) of the daily zonal wind at $60^{\circ} \mathrm{N}$ for DJF and at $60^{\circ} \mathrm{S}$ for SON between STDD and CONV shows that they are very close to each other (Fig. 4b, e). It is difficult to notice frequency differences of easterly winds, or MSSWs, in NH during DJF in Figs. $3 b$ and $4 b$, which are further examined in Sect. 3.3. In contrast, one can see that STDD shows SH easterly winds in late September, reflecting the occurrence of the MSSW in September 2002, whereas CONV underrepresents it as a minor SSW without a zonal wind reversal (Fig. 3d, e).

AMIP has stronger climatological winds from midwinter to spring and somewhat smaller variability around January in NH. One also sees in Fig. 3a, c that zonal wind reversals during DJF are less frequent for AMIP than for STDD. These features are reflected in PDFs of daily zonal wind data for STDD and AMIP (Fig. 4c). It is clear that the PDF for AMIP is biased toward the positive side, consistent with the stronger climatological westerly wind and less frequent zonal wind reversals. It is also notable that, apart from the climatological difference, the daily zonal wind data for AMIP has smaller variability. Another PDF is drawn in the thin solid line in Fig. 4c for zonal wind data for AMIP that are artificially decreased by the climatological wind difference between STDD and AMIP, so that this PDF has the same mean value as the STDD PDF. One sees that this PDF has somewhat lower frequencies of extreme (both strong and weak) wind values.

The AMIP zonal wind data are also biased toward stronger vortex states in the $\mathrm{SH}$ extratropical stratosphere for $\mathrm{SH}$ winter and spring (Figs. 3f and 4f). The stronger climatological wind is notable from September to November. No MSSW 
(a) STDD, DJF

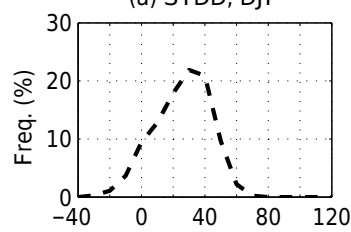

(d) STDD, SON

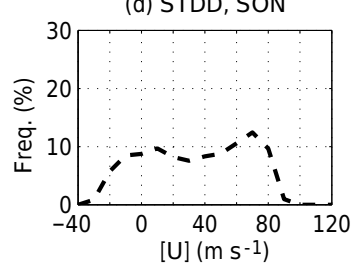

(b) CONV, DJF

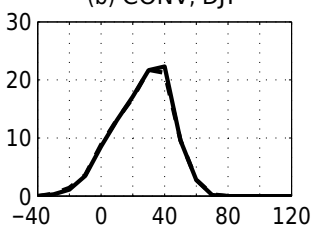

(e) CONV, SON

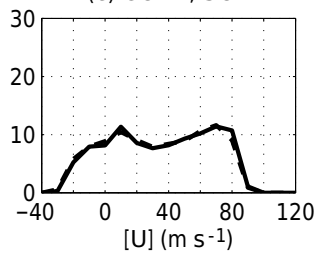

(c) AMIP, DJF

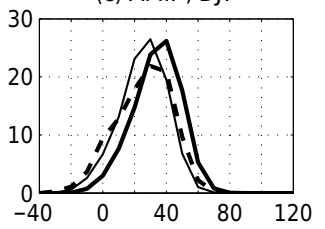

(f) AMIP, SON

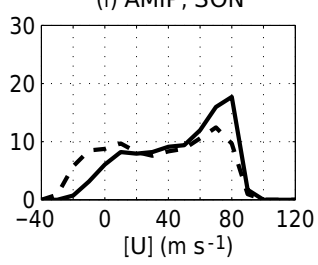

Figure 4. PDFs of the daily zonal mean zonal wind at $60^{\circ} \mathrm{N}, 10 \mathrm{hPa}$, for DJF (a-c) and at $60^{\circ} \mathrm{S}, 10 \mathrm{hPa}$, for SON (d-f) in broken lines (a, d) or in solid lines (the other panels). Panels (a, d) are for STDD, (b, e) for CONV, and (e, f) for AMIP. Broken lines in (b, c, e, f) denote the results from STDD. The STDD data after the 1972-1973 season are used in (b, e). In panel (c), an additional PDF is drawn in the thin solid line for the AMIP zonal wind data that are artificially decreased by the climatological difference from STDD, so that the decreased wind data have the same mean value as in STDD.

(a) STDD

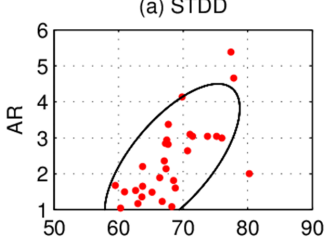

(d) STDD

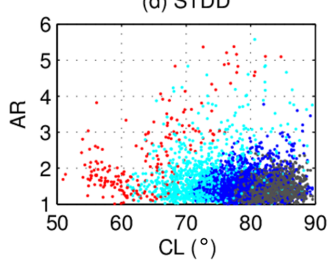

(b) CONV

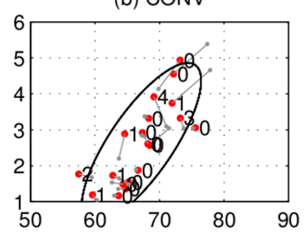

(e) CONV

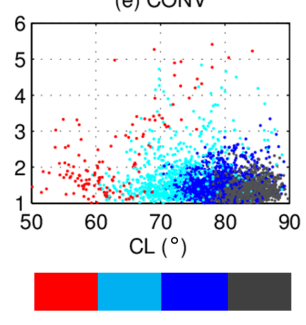

$\begin{array}{llll}0 & 20 & 40 & {[U]\left(\mathrm{m} \mathrm{s}^{-1}\right)}\end{array}$ (c) AMIP

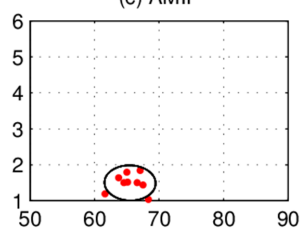

(f) AMIP

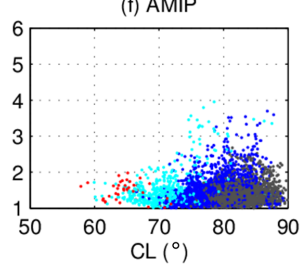

Figure 5. (a-c) Scatter plots between CL and AR of the $10 \mathrm{hPa}$ height on the onset dates of the NH MSSWs: (a) STDD, (b) CONV, and (c) AMIP. Ellipses denote representative distributions of the data points extracted by an empirical orthogonal function analysis. Each data point in (b) is connected to the corresponding case in STDD (gray) and accompanied by a number denoting the time difference in the onset dates (Table 1). Panels (d-f) plot results for all DJF days available to each product. Each data point is colored by the zonal mean zonal wind at $60^{\circ} \mathrm{N}, 10 \mathrm{hPa}$ (see the color bar).

(zonal wind reversal) is simulated from September to midOctober in AMIP.

\subsection{Frequency and vortex geometry of MSSWs in NH}

Figures 3 and 4 showed that the zonal wind variability in NH looks similar between STDD and CONV, suggesting that the occurrence of MSSWs is also similar. On the other hand, AMIP clearly lacks the zonal wind variability and easterly winds, which is suggestive of fewer MSSWs. In this subsection, we examine the frequency and also vortex geometry of NH MSSWs for the three products.
Table 1 lists the onset dates of DJF MSSWs identified for STDD and CONV. The frequency of DJF MSSWs for STDD is 30 events in the 56 seasons (53.6\% for each season) and 22 events in the 41 seasons $(53.7 \%)$ after 1972-1973. CONV shows 19 events in the 41 seasons $(46.3 \%)$. A comparison of the MSSW onset dates between STDD and CONV in the 41 seasons shows that CONV reproduces most MSSWs in STDD as inferred from Fig. 3 but delays seven cases by 1 to 4 days. CONV also misses three cases, underrepresenting them as minor SSWs that do not accompany a zonal wind reversal. The latter feature is similar to the SH MSSW in 
(a) $\mathrm{NH}$

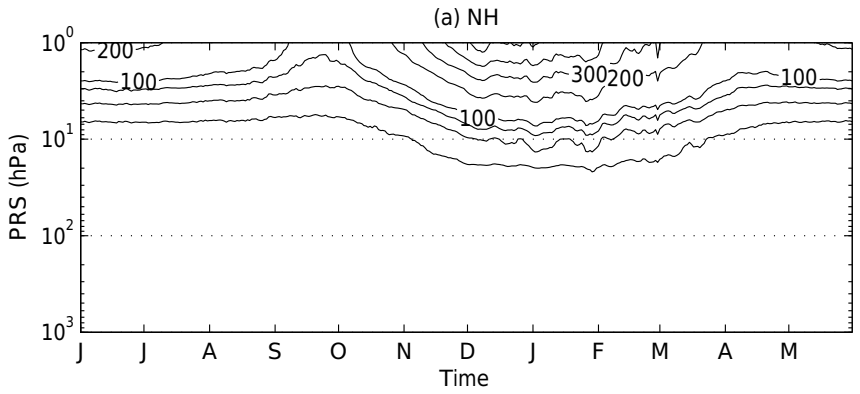

(c) $\mathrm{SH}$

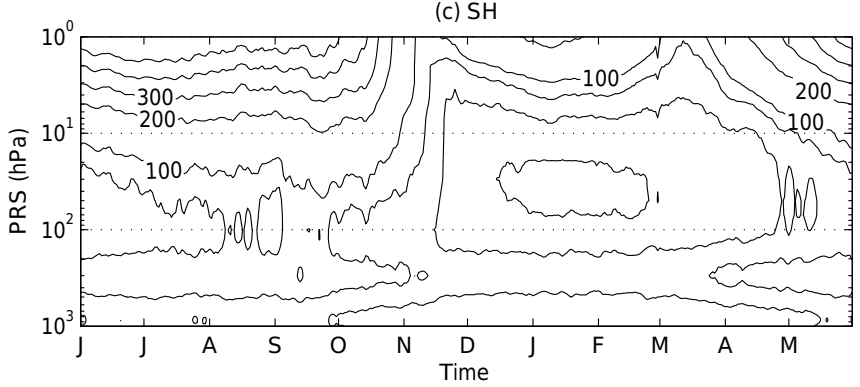

(b) $\mathrm{NH}, \mathrm{DJF}$
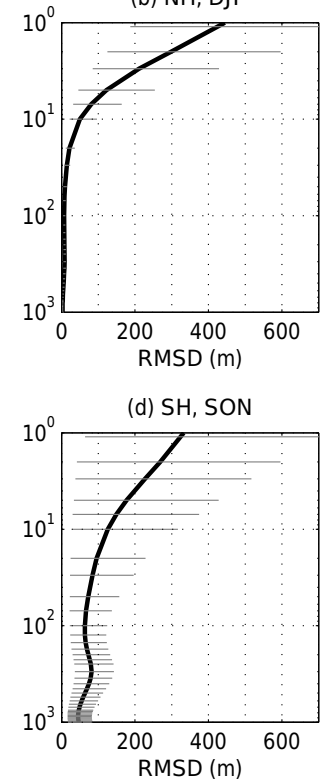

Figure 6. (a, c) Time-height sections of the climatology of RMSD: (a) NH and (c) SH. The contour interval is $100 \mathrm{~m}$, with additional contours at 25, 50, and $75 \mathrm{~m}$. Panels (b, d) plot seasonal means as a function of height: (b) NH for DJF and (d) SH for SON. The horizontal lines denote 5 th and 95 th percentiles.

September 2002, when CONV represents it as a minor SSW (Fig. 3). It is also noted that no opposite case exists where an onset date is represented earlier in CONV than in STDD or where an MSSW is represented only in CONV. The delayed and missed cases seem distributed randomly in time (year).

The frequency of DJF MSSWs for AMIP is 9 for the 56 seasons $(16.1 \%)$, which is much smaller than for STDD. The lower frequency of MSSWs in AMIP is consistent with the stronger westerly wind (Figs. 1-4).

The geometry of the polar vortex during the MSSWs for STDD is characterized in a scatter plot between CL and AR on lag $=0$ day (Fig. 5a). One sees that the data points for STDD roughly form a linear distribution, with a correlation coefficient of +0.68 . Data points located near the lower-left end correspond to MSSWs of low CL (e.g., vortex displacement MSSWs), and those near the opposite end correspond to MSSWs of high AR (e.g., vortex-split MSSWs). Such a linear distribution was pointed out by Taguchi (2016). This distribution reflects that the zonal mean zonal wind at $60^{\circ} \mathrm{N}$, $10 \mathrm{hPa}$, representing the vortex strength tends to weaken as CL decreases and/or AR increases (Fig. 5d).

CONV roughly reproduces a similar linear distribution of $\mathrm{CL}$ and AR on the MSSW onset dates. The differences in CL and AR vary from one MSSW to another.

In addition to the lower frequency, the MSSWs for AMIP show a notable feature about the vortex geometry - that all MSSWs have low CL and AR. No MSSW in AMIP shows high AR, e.g., over 2 , on lag $=0$ day. This implies a lack of vortex-split MSSWs. The absence of MSSWs of high AR is consistent with the scatter plot for all DJF data for AMIP (Fig. 5f): the data points for AMIP are biased toward high $\mathrm{CL}$ and low AR.

In the following, we further explore these differences in the frequency and vortex geometry of MSSWs. In Sect. 4, we relate the occurrence of MSSWs to general RMSD distributions of geopotential height fields between STDD and CONV. In Sect. 5, we examine strong planetary wave forcings and associated stratospheric vortex weakenings for STDD and AMIP.

\section{Comparison of CONV to STDD}

In Sect. 3, we showed that CONV is close to STDD in terms of the climatological zonal mean zonal wind and height in the NH extratropical stratosphere (Figs. 1 and 2). The daily time series and PDFs of the zonal wind also look similar between the two runs (Figs. 3 and 4), although the occurrence of MSSWs is slightly different. In this section, we describe general RMSD distributions of daily extratropical height fields between STDD and CONV at various levels and relate them to the occurrence of MSSWs.

\subsection{Climatological RMSD distributions}

First, we examine climatological distributions of RMSD for $\mathrm{NH}$ and SH. Figure 6a, c plot the long-term means of RMSD as a function of time (season) and height. It is common between the two hemispheres and all seasons for RMSD to in- 
(a) $\mathrm{NH}$

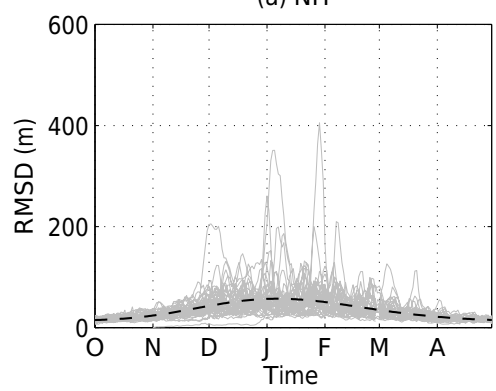

(c) $\mathrm{NH}, \mathrm{DJF}$

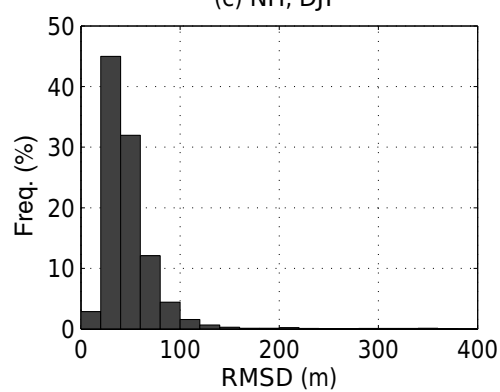

(b) $\mathrm{SH}$

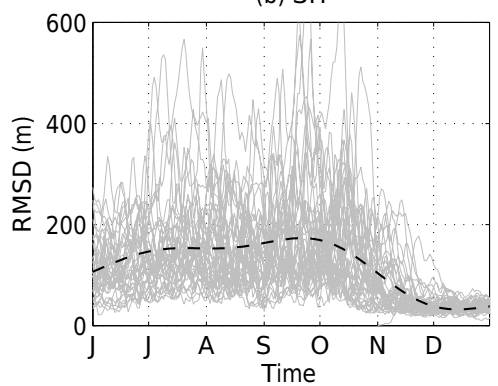

(d) $\mathrm{SH}, \mathrm{SON}$

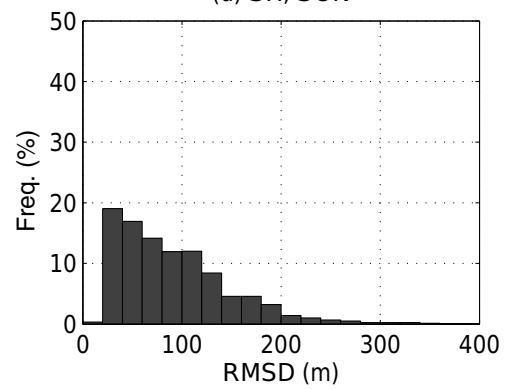

Figure 7. (a, b) Daily time series of RMSD at $10 \mathrm{hPa}$ (a) NH and (b) SH. Broken lines denote the climatological seasonal cycle. Panels (c, d) show PDFs of the RMSD values for DJF in NH and for SON in SH.

crease with height. This result will be expected, since the assimilation of conventional observations in CONV does not extend to the upper stratosphere. In the NH upper stratosphere, RMSD has a semiannual cycle, with two peaks in $\mathrm{NH}$ summer and winter. The winter peak is larger than the summer counterpart and extends deeper down to the middle stratosphere, such as $10 \mathrm{hPa}$. Such a semiannual cycle is also notable in the SH upper stratosphere. The winter peak also extends deeper than the summer peak in $\mathrm{SH}$ as in $\mathrm{NH}$.

The climatological height distributions of RMSD for NH winter and SH spring are extracted in Fig. 6b, $d$ to emphasize interhemispheric differences during the dynamically active seasons. It confirms the increase in RMSD with height for both hemispheres. It also reveals that RMSD is larger in SH than in NH in a large part of the domain, except for the upper stratosphere. The SH has considerable magnitudes of RMSD even in the troposphere. It may be that conventional observations are insufficient in $\mathrm{SH}$, and assimilation of satellite observations lead to larger differences.

An examination of year-to-year changes in RMSD suggests a trend in $\mathrm{SH}$, which is discussed in Sect. 6.2.

\subsection{Case-to-case variability in RMSD and MSSWs in NH}

We further examine day-to-day variability in RMSD, particularly for cases of extreme RMSD values, and compare it to the occurrence of MSSWs. Figure 7a, b show daily time series of RMSD at $10 \mathrm{hPa}$ in both $\mathrm{NH}$ and $\mathrm{SH}$ during dynamically active seasons. PDFs of the RMSD values are also plotted in Fig. 6c, d: DJF and SON data are used for $\mathrm{NH}$ and $\mathrm{SH}$, respectively. In addition to the climatological difference, notable minimum and maximum RMSD values are also larger in SH than in NH. SH RMSD often attains large local maxima, e.g., over $400 \mathrm{~m}$, and even NH RMSD sometimes exhibits sharp peaks.

In order to examine specific geopotential height distributions, we identify cases of extremely large RMSD values for NH and SH as when RMSD attains local maxima over a threshold. The threshold is defined as the 95th percentile of all RMSD values during DJF in NH or SON in $\mathrm{SH}$ for the 41 seasons. The local maxima must be separated by more than 30 days when they are identified in the same season. RMSD values around the MSSW onset dates (from lag $=-10$ to +10 days) are excluded from this procedure and are used separately.

Figure 8 presents scatter plots of the zonal and wave components contributing to the total RMSD values for these cases. Note that the distance of each data point from the origin gives the total RMSD value (Eq. 2). The figure also includes the results of all MSSW onset dates identified in STDD. The data points use different colors according to the zonal mean zonal wind at $60^{\circ} \mathrm{N} / \mathrm{S}, 10 \mathrm{hPa}$, in STDD on the target day of each case (see the color bar). Note that data points plotted in red corresponds to MSSWs in STDD.

One sees in Fig. 8a that several data points are located far from the origin and hence have the largest total RMSD values. These cases, except for one MSSW, occur separately from the MSSWs. Some of the data points are located relatively close to the $y$ axis, implying large contributions from 
(a) $\mathrm{NH}$

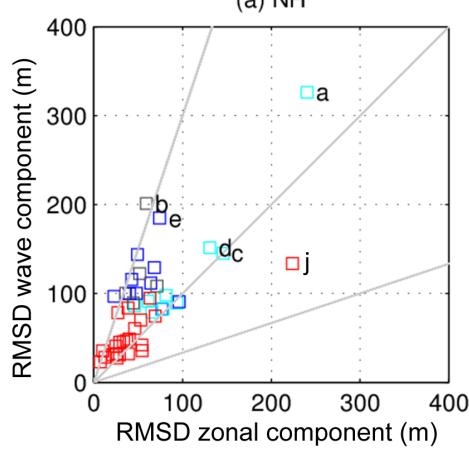

(c) $\mathrm{NH}$ (larger version)

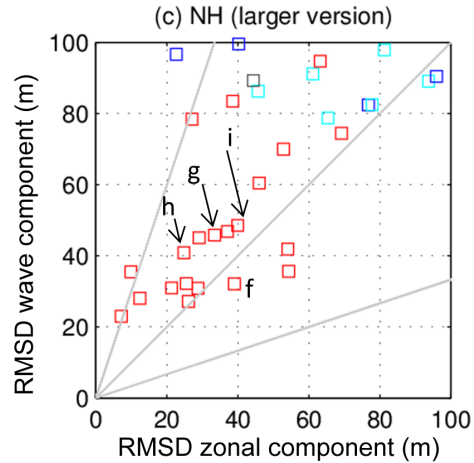

(b) $\mathrm{SH}$

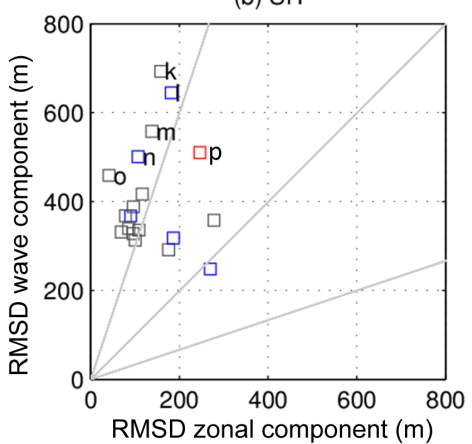

[U] STDD $\left(\mathrm{m} \mathrm{s}^{-1}\right)$

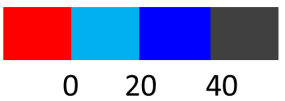

Figure 8. (a, b) Scatter plots between zonal and wave contributions to total RMSD for large RMSD peaks exceeding the 95th percentile of all RMSD values: (a) NH for DJF and (b) SH for SON. Results for all MSSW onset dates in STDD are also shown. Panel (c) is a larger version of (a). The data points are plotted in different colors, which denote the zonal mean zonal wind in STDD at $60^{\circ} \mathrm{N} / \mathrm{S}, 10 \mathrm{hPa}$, on the target dates (see the color bar). Red data points correspond to the MSSW cases. Letters a-j in (a, c) and k-p in (b) denote the cases used in Fig. 9. Gray lines denote $y=x / 3, y=x$, and $y=3 x$.

the wave component. The zonal mean zonal wind in STDD is relatively strong (over $20 \mathrm{~m} \mathrm{~s}^{-1}$ ) for these cases when they have large values of the $y$ component $(100 \mathrm{~m})$.

The opposite also holds for other data points, when the zonal component makes large contributions and the zonal wind is weaker $\left(20 \mathrm{~m} \mathrm{~s}^{-1}\right)$. We also examined zonal wind differences of CONV from STDD for these cases but did not notice any systematic relationship, as both overestimations and underestimations by CONV occur (not shown). The five largest values of total RMSD are labeled as a-e in Fig. 8a for Fig. 9.

Regarding the MSSWs, one may expect that the missed and delayed cases have larger RMSD values than the other MSSWs, but it turns out that this is not the case (see also Fig. 8c). All of the three missed cases (labeled as $\mathrm{f}-\mathrm{h}$ ) and one of the delayed cases (with a time difference of 4 days, labeled as i) appear in the intermediate data points near the coordinate point of $(40,40)$. The exceptional MSSW, located near $(200,100)$, is a delayed case with a time difference of 3 days (labeled as $\mathrm{j}$ ).

As for $\mathrm{SH}$, a large part of the data points are located near the $y$ axis, implying an important role of the wave component. For these cases, STDD has strong westerly winds over $20 \mathrm{~m} \mathrm{~s}^{-1}$, and both overestimations and underestimations in the zonal wind by CONV occur. The missed MSSW case (September $2002 \mathrm{MSSW}$ in STDD, labeled as p) also occurs with a large contribution from the wave component.

Figure 9a-e plot $10 \mathrm{hPa}$ height maps for the five largest RMSD values in NH selected from Fig. 8a. In these figure panels, we see large differences in the $10 \mathrm{hPa}$ height occurring in different (or various) locations depending on the target cases, which often occur near the edge of the polar vortex rather than near the center of the vortex. These differences do not have strong zonal symmetry but vary in the zonal direction, consistent with the large contributions from the wave component (Fig. 8a). These differences are reflected in different distributions of contour lines of $30000 \mathrm{~m}$ representative of the vortex edge, implying different shapes of the vortex between STDD and CONV.

The height differences during the four MSSWs (three missed cases and one delayed case, Fig. 9f-i) are generally smaller in magnitude. Both STDD and CONV show very similar vortex shapes in terms of the $30000 \mathrm{~m}$ contour lines. The difference fields have negative, albeit small in magnitude, values over polar latitudes, suggesting that the extratropical zonal wind is stronger in CONV. This is consistent with the fact that these MSSWs are missed or delayed in CONV. 

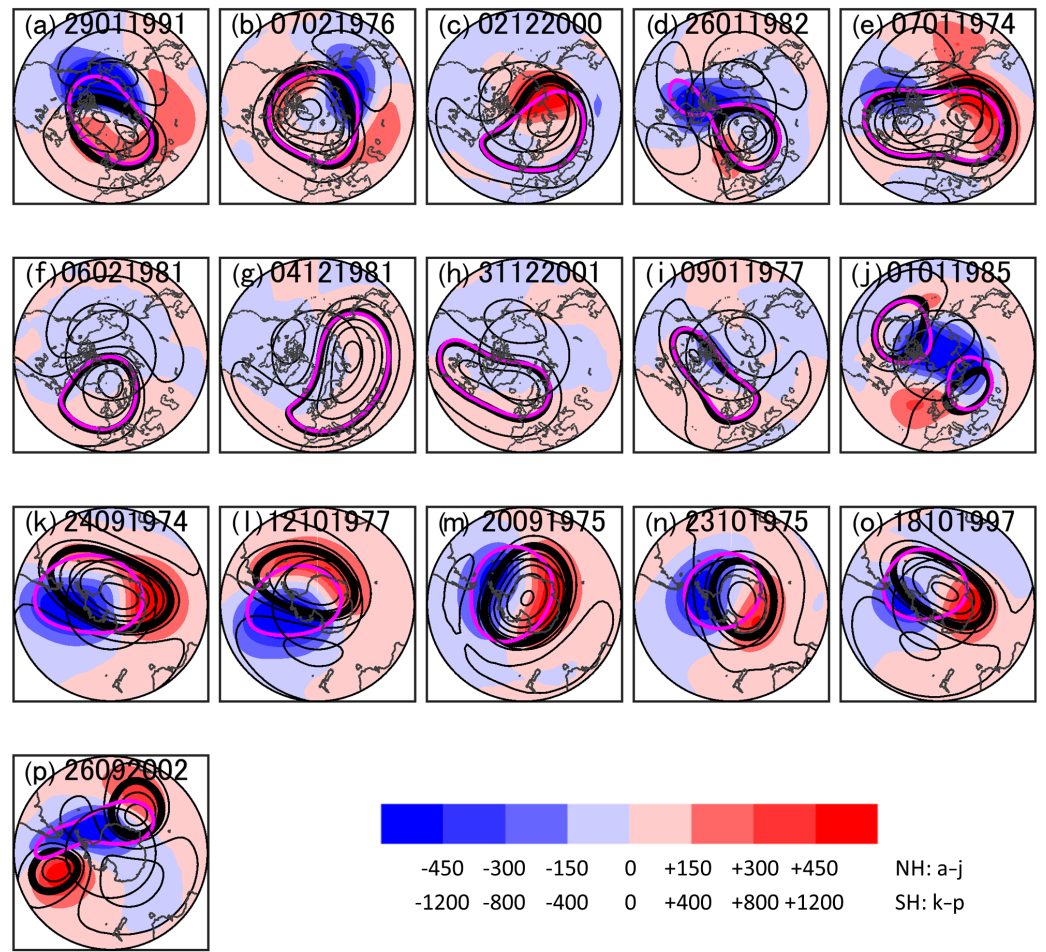

Figure 9. (Black contours in (a-e) and (k-o).) Maps of the $10 \mathrm{hPa}$ height in STDD for the five largest RMSD values: (a-e) NH and (k-o) SH. These labels are the same as in Fig. 8a, b. The target dates are also denoted in the ddmmyyyy format. The contour interval is $500 \mathrm{~m}$, and thick black contours denote $30000 \mathrm{~m}$ in STDD. Magenta contours denote $30000 \mathrm{~m}$ for the $10 \mathrm{hPa}$ height in CONV on the same dates. Color shades denote differences of CONV from STDD (see the color bar). Panels (f-j) are similar, but for the three missed MSSW cases and the two delayed cases in NH. These labels are also denoted in Fig. 8a, c. Panel (p) is similar, but for the 2002 MSSW in SH (see Fig. 8b).

One exception is the MSSW with the time difference of 3 days (Fig. 9j). In this case, the polar vortex similarly splits into two cyclones for both STDD and CONV. Nonetheless, the height differences have largely negative values at high latitudes.

Figure $9 \mathrm{k}-\mathrm{o}$ are similar, but for the $\mathrm{SH}$ cases of the five largest RMSD values (Fig. 8b). The differences in SH are generally larger in magnitude than the $\mathrm{NH}$ counterparts and are dominated by the wave 1 component. The dominance of wave 1 reflects that the location of the polar vortex (or distribution of the $30000 \mathrm{~m}$ contour) in CONV is notably deviated from the STDD counterpart.

As for the SH MSSW, STDD shows a split of the vortex into two cyclones, whereas CONV shows that the vortex (30000 m contour line) shifts away from the South Pole and highly stretches, without a split (Fig. 9p). The height differences are comparable in magnitude to the preceding five cases. Negative values prevail in the height difference over polar latitudes, consistent with the underrepresentation of this case as a minor SSW in CONV.

\section{Comparison of AMIP to STDD}

We showed in Sect. 3 that the MSSW frequency for AMIP is much lower than that for STDD. In particular, AMIP lacks MSSWs of high AR. In order to better understand these differences (especially the former), in this section we examine extreme planetary wave forcings (poleward eddy heat fluxes) and stratospheric vortex weakening responses to them. Since it is well known that MSSWs are a response of the polar vortex to anomalously strong planetary wave forcings from the troposphere (Matsuno, 1971; Limpasuvan et al., 2004), the differences may be explained in terms of wave forcing from the troposphere and/or the vortex response in the stratosphere.

\subsection{Lower MSSW frequency for AMIP}

The first issue is the lower frequency of MSSWs in AMIP. One may hypothesize that it is due to a lower frequency of strong wave forcings and/or weaker vortex responses to such wave forcings.

In Fig. 10a, we examine how the poleward eddy heat flux in the extratropical lower stratosphere $\left(40-90^{\circ} \mathrm{N}, 100 \mathrm{hPa}\right)$ and zonal wind deceleration are related for all MSSWs in 
(a) MSSWs

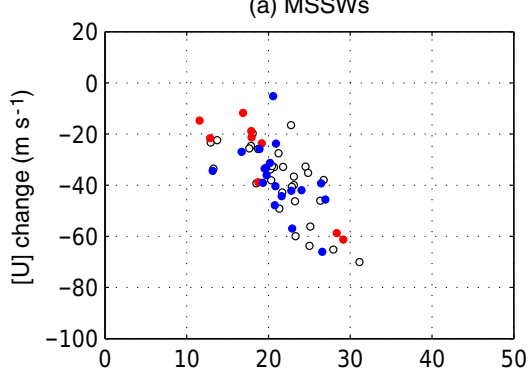

(c)

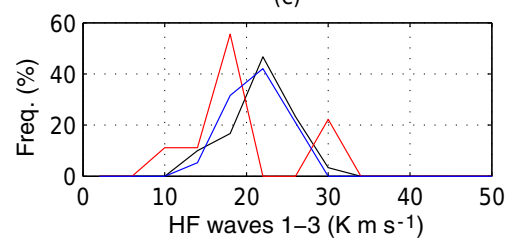

(b) MAX HF waves 1-3

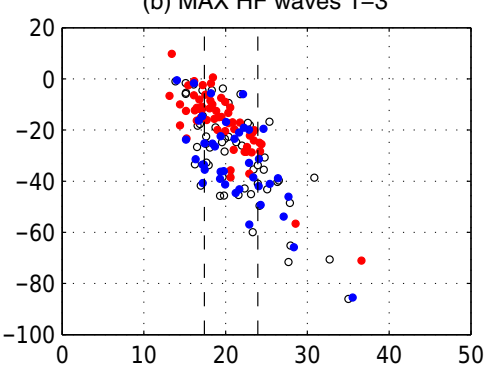

(d)

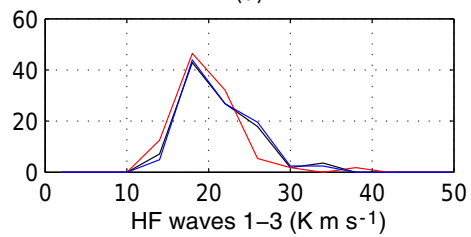

Figure 10. (a) Scatter plot between 21-day mean heat flux (lag $=-20$ to 0 day) of waves 1-3 and associated zonal wind deceleration for the 21 days for all MSSWs in STDD (black), CONV (blue), and AMIP (red). Panel (b) is similar but uses the maximum of the 21-day mean heat flux of waves 1-3 in each DJF season as the key. The vertical broken lines denote the 25th and 75th percentile values of the heat flux in STDD, which is used for the composite analysis in Fig. 11. Panels (c, d) show frequency distributions of the 21-day mean heat flux for the respective cases. The full period data are used for STDD.

STDD and AMIP. The heat flux is averaged in time from lag $=-20$ to 0 day, and the zonal wind deceleration is also calculated for the 21 days. We focus on the 21 day period, since the correlation between the two quantities for the MSSWs in STDD maximizes when we average the heat flux for about 10 to 30 days (not shown). This is consistent with the fact that polar vortex strength is highly correlated to the heat flux when the latter is averaged for a few weeks or longer (Polvani and Waugh, 2004). This method follows Taguchi (2017), who examined the Coupled Model Intercomparison Project Phase 5 (CMIP5) historical simulations with 30 models. It is common between STDD and AMIP that, as expected, the zonal wind decelerates more strongly when the wave forcing is stronger. The distribution for STDD has a correlation coefficient of -0.70 .

Since this plot is based on the data around the MSSWs, it does not explain how or why the MSSWs are much lower for AMIP. In order to obtain a clue for the difference in the MSSW frequency, we look at extreme planetary wave forcings by identifying a maximum of the poleward heat flux (averaged for 21 days, from 20 day before each day) of waves 1-3 for each winter season in STDD and AMIP. We also extract zonal wind decelerations associated with the maximum wave forcings (Fig. 10b).

The plot shows two features in addition to similar linear distributions between the wave forcing and wind deceleration for both STDD and AMIP as in the MSSWs. First, AMIP lacks relatively strong forcings. For example, STDD has 10 samples over a threshold of $25 \mathrm{~K} \mathrm{~m} \mathrm{~s}^{-1}$, whereas AMIP has only 3 . Second, the zonal wind decelerations for wave forc- ings around $20 \mathrm{~K} \mathrm{~m} \mathrm{~s}^{-1}$ seem stronger for STDD than for AMIP.

The second point is further examined in a composite analysis with respect to the days of the maximum heat flux that ranges from the 25 th percentile $\left(17.4 \mathrm{~K} \mathrm{~m} \mathrm{~s}^{-1}\right)$ to the 75 th percentile $\left(23.9 \mathrm{~K} \mathrm{~m} \mathrm{~s}^{-1}\right)$ of all maximum heat flux values in STDD (Fig. 11). The two percentile values are similar for AMIP: 16.9 and $22.0 \mathrm{~K} \mathrm{~m} \mathrm{~s}^{-1}$. The range limitation intends to subsample wave forcings of similar magnitudes for both STDD and AMIP.

As a result, the composite daily heat flux increases from lag $\approx-20$ to 0 day for both STDD and AMIP (Fig. 11c). The lag $=0$ day here denotes when the 21-day mean heat flux maximizes. This feature is quite similar between the two products by construction. The 21-day means from lag $=-20$ to 0 day are not significantly different at the $95 \%$ level. The composite residual mean meridional wind (in the transformed Eulerian-mean equations) and wave driving (EP flux divergence-convergence) are also similar between the two (Fig. 11b, d), and their 21-day means are not significantly different at the $95 \%$ level. In contrast, the composite zonal wind evolution is different, as the zonal wind decelerates more strongly for STDD. It is around $40 \mathrm{~m} \mathrm{~s}^{-1}$ before lag $\approx-20$ days in both STDD and AMIP, before decreasing to lag $\approx 0$ day. The wind deceleration from lag $=-20$ to 0 day is $28.5 \mathrm{~m} \mathrm{~s}^{-1}$ for STDD and $17.6 \mathrm{~m} \mathrm{~s}^{-1}$ for AMIP, and this difference is judged to be significantly different at the $95 \%$ level. This feature is consistent with Fig. 10b.

Thus, these results suggest that the lower frequency of MSSWs for AMIP can be attributed to the lower frequency of extreme wave forcings and the weaker vortex response in 
(a)

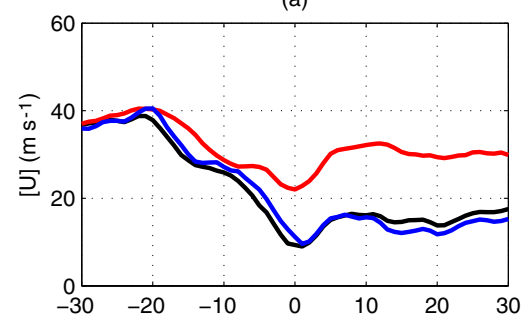

(b)

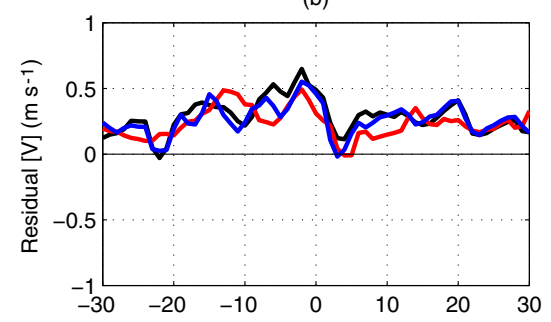

(c)

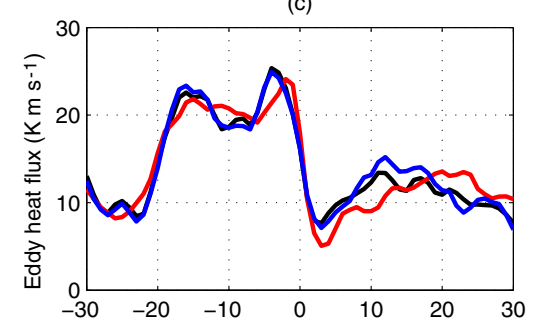

(d)

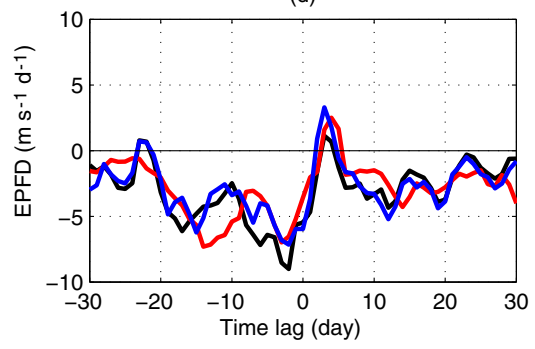

Figure 11. Composite time series with respect to the maximum of the 21-day mean heat flux of waves 1-3 in each DJF season for STDD (black), CONV (blue), and AMIP (red): (a) zonal mean zonal wind at $60^{\circ} \mathrm{N}, 10 \mathrm{hPa}$, (b) residual mean meridional wind in the transformed Eulerian-mean equations at $60^{\circ} \mathrm{N}, 10 \mathrm{hPa}$, (c) heat flux of waves $1-3$ at $40-90^{\circ} \mathrm{N}, 100 \mathrm{hPa}$, and (d) EP flux divergence-convergence (EPFD) of waves $1-3$ at $60^{\circ} \mathrm{N}, 10 \mathrm{hPa}$. The lag $=0$ day denotes when the 21-day mean heat flux maximizes. The full period data are used for STDD.

the stratosphere. These differences may reflect model biases in AMIP (or effects of data assimilation in STDD), which will be discussed in Sect. 6 .

Figures 10 and 11 additionally include results from CONV for reference. It is shown that the results from CONV are similar to those from STDD in terms of planetary wave forcing from the troposphere and vortex response in the stratosphere.

\subsection{Lack of MSSWs of high AR in AMIP}

The second issue is the lack of MSSWs of high AR in AMIP. Again, this may be due to a lack of appropriate wave forcings leading to such MSSWs and/or a difference in vortex responses to such forcings.

An analysis of AR changes associated with the maximum 21-day mean heat flux of waves 1-3 (defined for Figs. 10b, $\mathrm{d}$ and 11) in STDD and AMIP indicates that the lack of MSSWs of high AR in AMIP can be explained by a different vortex response in the stratosphere to strong wave forcings from the troposphere. The heat flux maxima of waves 1-3 include similar contributions from wave 1 and wave 2 between STDD and AMIP, but extreme AR values occur only for STDD (not shown).

It is noted that the climatological, or background, difference in the $10 \mathrm{hPa}$ height will play a role in explaining the different vortex response. The climatological difference is negative and increases in magnitude toward polar latitudes (Fig. 2). Therefore, it is likely to follow that when the polar vortex in STDD largely stretches for a high AR value in response to a wave forcing, it will be more difficult for the deeper AMIP vortex to exhibit similar stretching even for the same forcing. This idea is related to Seviour et al. (2016), who pointed out a close connection between the frequency of vortex-split MSSWs and climatological ARs for 13 CMIP5 models.

\section{Summary and discussion}

\subsection{Summary}

This study has compared large-scale dynamical variability in the NH and SH extratropical stratosphere, such as MSSWs, among the JRA-55 family data sets. In spite of the importance of dynamical variability in the stratosphere, such an aspect was relatively unexplored in the JRA-55 family data sets and also in other reanalyses. This study owes the comparison to the meticulous design of the JRA-55 family data sets.

First, a survey of the climatological states confirms the stronger polar night jet in both hemispheres for CONV and AMIP than for STDD. This difference is more notable for AMIP. A comparison of MSSWs reveals that CONV reproduces a large part of MSSWs identified in STDD. However, CONV delays several cases by 1 to 4 days and also misses three cases, underrepresenting them as minor SSWs. The SH MSSW in September 2002 is also underrepresented as a minor SSW in CONV. AMIP lacks MSSWs, especially those of high AR values. The differences in CONV could be understood by the bias of the numerical weather prediction model (as seen in AMIP) and the paucity of data assimilation as hypothesized by Kobayashi et al. (2014). It is also suggested that, due to the bias of the model, even STDD (or CONV) 
(a) $1 \mathrm{hPa}$

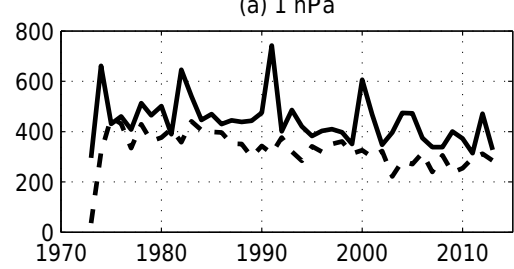

(c) $100 \mathrm{hPa}$

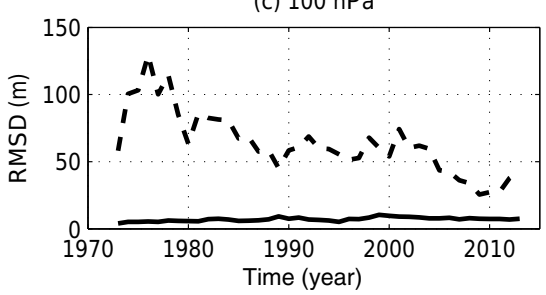

(b) $10 \mathrm{hPa}$

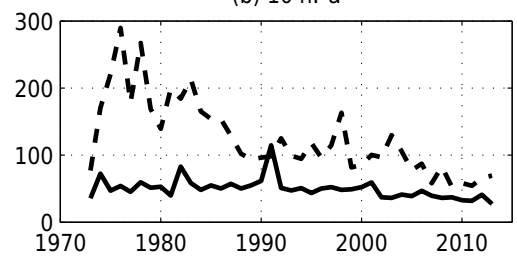

(d) $500 \mathrm{hPa}$

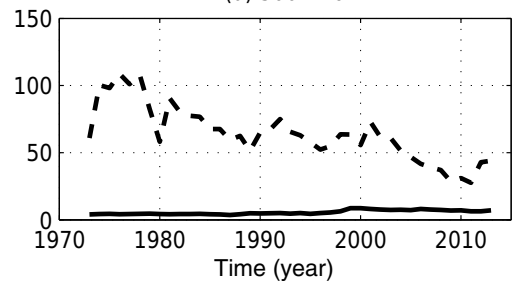

Figure 12. Year-to-year variations of RMSD at four levels as indicated. Solid lines denote results in NH for DJF, and broken lines denote results in $\mathrm{SH}$ for $\mathrm{SON}$.

may underestimate stratospheric variability compared to the real world.

Next, we examined daily RMSD distributions of geopotential height fields between STDD and CONV and relates them to the occurrence of MSSWs. In spite of the slight climatological differences in the zonal mean zonal wind and $10 \mathrm{hPa}$ geopotential height, the examination shows that CONV is sometimes significantly different from STDD in terms of daily height fields. The differences are especially large in the upper stratosphere in both hemispheres during dynamically active seasons, with larger values in $\mathrm{SH}$. The RMSD values in NH winter sometimes have sharp peaks, apart from the MSSWs. The RMSD peaks include contributions from the wave component that are larger than or comparable to those from the zonal component. RMSD values for some of the delayed MSSWs and all of the missed MSSWs are smaller than such peaks. In such MSSW cases, CONV overestimates the zonal mean zonal wind in the extratropical stratosphere by definition, and both of the zonal and wave components make important contributions.

Furthermore, we examined strong planetary wave forcings and associated zonal wind decelerations (polar vortex weakenings) in STDD and AMIP. It turns out that in AMIP, extreme wave forcings are fewer and zonal wind decelerations to such wave forcings are weaker. The stronger polar vortex in AMIP may also contribute to the lack of MSSWs since it will act to prohibit "preconditioning" of the vortex for MSSWs. These features are commonly seen in some of the CMIP5 historical simulations (Taguchi, 2017). These model biases may be reflected in CONV, where they are likely to contribute to the delay and miss several MSSWs. The latter factor (different vortex responses in AMIP from STDD) is also suggested to be relevant to the lack of MSSWs of high AR values in AMIP.

\subsection{Discussion}

Finally, we discuss several aspects unanswered in this study.

This study reveals large RMSD values for stratospheric height fields between STDD and CONV (Figs. 7-9), but it does not fully answer how or why such differences occur. It does not seem simple to understand what patterns such large differences take and when they occur. They could be explained by the effects of the assimilation of satellite data, but future study is needed to understand the effects more specifically.

Whereas we mostly focus on the middle stratosphere, such as $10 \mathrm{hPa}$ height distributions, the RMSD increase with height suggests that upper stratospheric heights have larger differences between STDD and CONV. This will invite a further study about differences in vertical vortex structures between the two products. For example, it is possible that even MSSWs identified on the same onset dates in STDD and CONV have notable differences in the upper stratosphere between them.

A further examination of year-to-year variations in RMSD at several levels suggests a decreasing trend in SH (Fig. 12). The SH decreasing trend is statistically significant at the $95 \%$ level for all four levels. Such a trend is absent or much weaker in $\mathrm{NH}$. This suggests that the impacts of assimilation of satellite data decrease over the decadal timescale especially in SH. Further details and mechanisms will be worthy of investigation.

The differences between STDD and AMIP, which are relevant to model biases, are characterized by the stronger climatological polar vortex and lack of MSSWs for AMIP. The lack of MSSWs in AMIP is not fully explained by the climatological difference, although it is consistent. This suggests that some additional factors or processes, such as tro- 
pospheric planetary wave forcing and stratospheric vortex response, may play a role.

Detailed analyses of these aspects will therefore be useful. A decomposition technique of eddy heat flux (e.g., Nishii et al., 2009; Fletcher and Kushner, 2011) could be applied so as to better understand the lack of extreme wave forcings from the troposphere in AMIP. A zonal momentum budget analysis as in Martineau et al. (2016) could also be conducted to diagnose stratospheric vortex responses to strong wave forcings in the three products, including how or why several MSSWs are delayed or missed in CONV compared to STDD.

Data availability. The JRA-55 family data used in this study are available at several data servers (on registration) including the Japan Meteorological Agency Data Dissemination System, see http://jra. kishou.go.jp/JRA-55/index_en.html.

Competing interests. The author declares that he has no conflict of interest.

Special issue statement. This article is part of the special issue "The SPARC Reanalysis Intercomparison Project (S-RIP) (ACP/ESSD inter-journal SI)". It is not associated with a conference.

Acknowledgements. The author thanks the Japan Meteorological Agency for making the JRA-55 family data sets available. The data were obtained from the Research Data Archive at the National Center for Atmospheric Research, Computational and Information Systems Laboratory. The author acknowledges that comments from three anonymous reviewers and Patrick Martineau improved the manuscript. This study is supported by the Grant-in-Aid for Scientific Research (S) 24224011 and (C) 15K05286.

Edited by: Peter Haynes

Reviewed by: three anonymous referees

\section{References}

Andrews, D. G., Holton, J. R., and Leovy, C. B.: Middle Atmosphere Dynamics, Academic Press, San Diego, California, 1987.

Butler, A. H., Seidel, D. J., Hardiman, S. C., Butchart, N., Birner, T., and Match, A.: Defining sudden stratospheric warmings, B. Am. Meteorol. Soc., 96, 1913-1928, https://doi.org/10.1175/BAMSD-13-00173.1, 2015.

Butler, A. H., Sjoberg, J. P., Seidel, D. J., and Rosenlof, K. H.: A sudden stratospheric warming compendium, Earth Syst. Sci. Data, 9, 63-76, https://doi.org/10.5194/essd-9-63-2017, 2017.

Charlton, A. J. and Polvani, L. M.: A new look at stratospheric sudden warmings. Part I: Climatology and modeling benchmarks,
J. Climate, 20, 449-469, https://doi.org/10.1175/JCLI3996.1, 2007.

Fletcher, C. G. and Kushner, P. J.: The role of linear interference in the annular mode response to tropical SST forcing, J. Climate, 24, 778-794, https://doi.org/10.1175/2010JCLI3735.1, 2011.

Fujiwara, M., Wright, J. S., Manney, G. L., Gray, L. J., Anstey, J., Birner, T., Davis, S., Gerber, E. P., Harvey, V. L., Hegglin, M. I., Homeyer, C. R., Knox, J. A., Krüger, K., Lambert, A., Long, C. S., Martineau, P., Molod, A., Monge-Sanz, B. M., Santee, M. L., Tegtmeier, S., Chabrillat, S., Tan, D. G. H., Jackson, D. R., Polavarapu, S., Compo, G. P., Dragani, R., Ebisuzaki, W., Harada, Y., Kobayashi, C., McCarty, W., Onogi, K., Pawson, S., Simmons, A., Wargan, K., Whitaker, J. S., and Zou, C.-Z.: Introduction to the SPARC Reanalysis Intercomparison Project (S-RIP) and overview of the reanalysis systems, Atmos. Chem. Phys., 17, 1417-1452, https://doi.org/10.5194/acp17-1417-2017, 2017.

Kobayashi, C. and Iwasaki, T.: Brewer-Dobson circulation diagnosed from JRA-55, J. Geophys. Res.-Atmos., 121, 1493-1510, https://doi.org/10.1002/2015JD023476, 2016.

Kobayashi, C., Endo, H., Ota, Y., Kobayashi, S., Onoda, H., Harada, Y., Onogi, K., and Kamahori, H.: Preliminary results of the JRA-55C, an atmospheric reanalysis assimilating conventional observations only, SOLA, 10, 78-82, https://doi.org/10.2151/sola.2014-016, 2014.

Kobayashi, S., Ota, Y., Harada, Y., Ebita, A., Moriya, M., Onoda, H., Onogi, K., Kamahori, H., Kobayashi, C., Endo, H., Miyaoka, K., and Takahashi, K.: The JRA-55 Reanalysis: General specifications and basic characteristics, J. Meteorol. Soc. Jpn. Ser. II, 93, 5-48, https://doi.org/10.2151/jmsj.2015-001, 2015.

Labitzke, K. G. and van Loon, H.: The Stratosphere: Phenomena, History, and Relevance, Springer, Berlin, 1999.

Limpasuvan, V., Thompson, D. W. J., and Hartmann, D. L.: The life cycle of the Northern Hemisphere sudden stratospheric warmings, J. Climate, 17, 2584-2596, https://doi.org/10.1175/15200442(2004)017<2584:TLCOTN>2.0.CO;2, 2004.

Manney, G. L., Allen, D. R., Krüger, K., Naujokat, B., Santee, M. L., Sabutis, J. L., Pawson, S., Swinbank, R., Randall, C. E., Simmons, A. J., and Long, C.: Diagnostic comparison of meteorological analyses during the 2002 Antarctic winter, Mon. Weather Rev., 133, 1261-1278, https://doi.org/10.1175/MWR2926.1, 2005.

Martineau, P. and Son, S.-W.: Quality of reanalysis data during stratospheric vortex weakening and intensification events, Geophys. Res. Lett., 37, 1-5, https://doi.org/10.1029/2010GL045237, 2010.

Martineau, P., Son, S.-W., and Taguchi, M.: Dynamical consistency of reanalysis data sets in the extratropical stratosphere, J. Climate, 29, 3057-3074, https://doi.org/10.1175/JCLI-D-150469.1, 2016.

Matsuno, T.: A dynamical model of the stratospheric sudden warming, J. Atmos. Sci., 28, 1479-1494, https://doi.org/10.1175/15200469(1971)028<1479:ADMOTS>2.0.CO;2, 1971.

Mitchell, D. M., Gray, L. J., Fujiwara, M., Hibino, T., Anstey, J. A., Ebisuzaki, W., Harada, Y., Long, C., Misios, S., Stott, P. A., and Tan, D.: Signatures of naturally induced variability in the atmosphere using multiple reanalysis datasets, Q. J. Roy. Meteor. Soc., 141, 2011-2031, https://doi.org/10.1002/qj.2492, 2015. 
Nishii, K., Nakamura, H., and Miyasaka, T.: Modulations in the planetary wave field induced by upward-propagating Rossby wave packets prior to stratospheric sudden warming events: A case-study, Q. J. Roy. Meteor. Soc., 135, 39-52, https://doi.org/10.1002/qj.359, 2009.

Polvani, L. M. and Waugh, D. W.: Upward wave activity flux as a precursor to extreme stratospheric events and subsequent anomalous surface weather regimes, J. Climate, $\quad 17, \quad 3548-3554, \quad$ https://doi.org/10.1175/15200442(2004)017<3548:UWAFAA>2.0.CO;2, 2004.

Seviour, W. J. M., Mitchell, D. M., and Gray, L. J.: A practical method to identify displaced and split stratospheric polar vortex events, Geophys. Res. Lett., 40, 5268-5273, https://doi.org/10.1002/grl.50927, 2013.

Seviour, W. J. M., Gray, L. J., and Mitchell, D. M.: Stratospheric polar vortex splits and displacements in the high-top CMIP5 climate models, J. Geophys. Res.-Atmos., 121, 14001413, https://doi.org/10.1002/2015JD024178, 2016.
Taguchi, M.: Connection of predictability of major stratospheric sudden warmings to polar vortex geometry, Atmos. Sci. Lett., 17, 33-38, https://doi.org/10.1002/asl.595, 2016.

Taguchi, M.: A study of different frequencies of major stratospheric sudden warmings in CMIP5 historical simulations, J. Geophys. Res., 122, 5144-5156, https://doi.org/10.1002/2016JD025826, 2017.

Waugh, D. W. and Polvani, L. M.: Stratospheric polar vortices, in: The Stratosphere: Dynamics, Transport, and Chemistry, edited by: Polvani, L. M., Sobel, A. H., and Waugh, D. W., American Geophysical Union, Washington, D.C., 43-57, 2010.

Yoden, S., Taguchi, M., and Naito, Y.: Numerical Studies on Time Variations of the Troposphere-Stratosphere Coupled System, J. Meteorol. Soc. Jpn., 80, 811-830, 2002. 